\title{
Placental Blood Drainage Shortens Duration of the Third Stage of Labor in Women Slowly Administered 20 IU Oxytocin
}

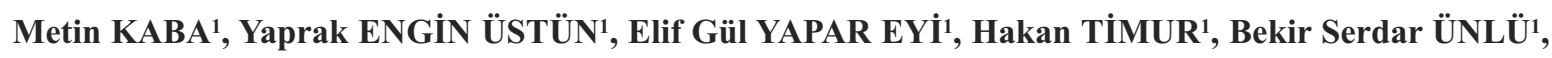 \\ Ayşegül ÖKSÜZOĞLU1
}

Ankara, Turkey

\section{ABSTRACT}

OBJECTIVE: Prolongation of the third stage of labor may cause serious postpartum complications. Thus, timely expulsion of the placenta is essential for preventing complications of the third stage of labor. We conducted a prospective cohort study to determine the effect of placental cord drainage on the duration of the TSL in women administered intravenous oxytocin during this stage.

STUDY DESIGN: This was a prospective cohort study in which 112 low risk pregnant women were allocated to the study. There were 53 women in the placental cord drainage group, and 59 women in the cord clamping group without drainage. Immediately after fetal delivery, intravenous infusion of 20 IU oxytocin in $500 \mathrm{~mL}$ Ringers' Lactate was started and infused within 2 hours in women in both groups. Outcome measures were the third stage duration and hemoglobin differences between admission and the postpartum sixth hour.

RESULTS: There were no significant differences between the two groups with regard to duration of stages 1 and 2 of labor, hemoglobin level on admission, and at the postpartum sixth hour, and hemoglobin differences between admission and the postpartum sixth hour. The median third stage duration in the study group was 3.40 (range: $0.35-16.20$ ) minutes, and 5.10 (range: $2.30-11.00$ ) minutes in the control group. This difference between the groups was statistically significant $(p<0.01)$.

CONCLUSION: Placental cord drainage reduces the third stage duration following vaginal deliveries in patients receiving intravenous oxytocin. Placental cord drainage is simple, does not require additional cost, and is applicable for every delivery.

Keywords: Third stage of labor, Active management of the third stage, Placental cord drainage, Oxytocin, Expectant management

\section{Gynecol Obstet Reprod Med 2017;23(1):6-10}

\section{Introduction}

The third stage of labor (TSL) is defined as the time interval between delivery of the baby and the expulsion of the pla-

${ }^{\prime}$ Department of Obstetrics and Gynecology Zekai Tahir Burak Women's Health Education and Research Hospital, Ankara

Address of Correspondence: Metin Kaba

Akdeniz University Faculty of Medicine

Department of Obstetrics and

Gynecology Antalya, Turkey metinkaba12@hotmail.com

Submitted for Publication: $\quad 11.07 .2016$

Accepted for Publication: $\quad 23.09 .2016$

:The abstract of this manuscript has been sent to $10^{\text {th }}$ Turkish Germany Gynecology Congress 2014 in Turkey.

\begin{tabular}{|c|c|}
\hline \multicolumn{2}{|c|}{ Access this article online } \\
\hline $\begin{array}{c}\text { Quick Response Code: } \\
\text { 口, }\end{array}$ & Website: www.gorm.com.tr \\
\hline 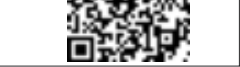 & DOI:10.21613/GORM.2016.612 \\
\hline
\end{tabular}

How to cite this article: Kaba M. Engin Üstün Y. Yapar Eyi EG. Timur H. Unlü BS. Oksüzoğlu A. Placental Blood Drainage Shortens Duration of the Third Stage of Labor in Women Slowly Administered 20 IU Oxytocin. Gynecol Obstet Reprod Med 2017;23(1):6-10 centa. After delivery of the baby, health care providers usually give attention to the newborn. But this stage is also important for the woman who gave birth. Prolongation of the TSL leads to an increased likelihood of maternal morbidity and mortality and TSL-related complications, which include uterine atony, retained placenta, postpartum hemorrhage (PPH), hemorrhagic shock, and even maternal death $(1,2)$. Reported childbirth-related maternal mortality is approximately 250.000 and 300.000 in the world annually (3). The majorities of these mortalities are caused by complications during the TSL, and most commonly are from uterine atony and PPH (3). The timely expulsion of the placenta and an efficient uterine contraction to cease bleeding are important parts of the TSL in preventing complications. Placental delivery is essential in allowing the uterus to contract and decrease blood loss during the TSL. Placental expulsion depends on the separation of the placenta from the uterine wall, capillary hemorrhage, uterine muscle contractile ability after placental separation, maternal effort, and gravity of the placenta $(4,5)$. Separation of the placenta from the uterine wall is usually completed within 15 
minutes in $90 \%$ of women after a fetal delivery $(2,6)$. Prolongation of the TSL increases the development of PPH, which is responsible for one quarter of maternal deaths globally, one third of all pregnancy-related deaths in Africa and Asia, and approximately 140.000 deaths annually $(7,8)$.

Preventive measures to decrease complications from the TSL are recommended for all women undergoing childbirth (9). Interventions to prevent TSL complications are called active management of the third stage of labor (AMTSL). These interventions include early umbilical cord clamping, administration of an oxytocic agent, and controlled cord traction until delivery of the placenta $(3,8)$. Placental cord drainage is also considered as an AMTS method. Placental cord drainage facilitates placental delivery in both vaginal and cesarean section deliveries $(10,11)$. Releasing the maternal side of the cord clamp and allowing the drainage of placental blood has been recommended by many obstetricians to facilitate the delivery of the placenta in vaginal deliveries (11).

We conducted this prospective cohort trial to determine whether placental cord drainage would shorten the TSL duration in low-risk pregnant women receiving 20 IU oxytocin in $500 \mathrm{~mL}$ Ringer's Lactate (RL) intravenous (IV) infusion within 2 hours.

\section{Material and Method}

This study was undertaken between 1 February and 30 June 2010 in the obstetric unit of our hospital. The study was approved by the Institutional Ethics Committee and performed in accordance with the Helsinki Declaration (Institutional ethical approval no: 19/04/2010/6).

On admission to the labor room, a detailed history, vaginal examination, and blood count estimation were carried out with all patients. Eligible patients were informed about the study and written informed consent was obtained from each patient. A total of 112 women who had a vaginal delivery completed the trial. There were $53(47.3 \%)$ women in the study group and $59(52.6 \%)$ in the control group. Inclusion criteria were singleton cephalic presentation pregnancies, patients willing to give written informed consent and pregnancies between 37 and 42 weeks of gestational age. Exclusion criteria were intrauterine fetal demise, antepartum hemorrhage, malpresentation, hypertensive disorders, history of medical disorders, multiple pregnancy, chorioamnionitis, and coagulation abnormalities. Fetal and maternal monitoring during the first and second stages of labor were carried out. In cases where augmentation or induction of labor was indicated, an oxytocin infusion protocol was applied.

When the cervix was dilated to $10 \mathrm{~cm}$ and the fetus arrived at the perineum, the pregnant woman was transferred to the delivery ward. Following delivery of the neonate, the umbilical cord was clamped with two clamps, cut between the clamps, and the neonate was handed to the nurse who was educated for neonatal care. In the placental cord drainage group, after the cord was clamped and cut, immediate unclamping was performed on the maternal side of the cord and the blood in the placenta was allowed to drain freely. In the control group, the umbilical cord remained clamped until placental delivery. Immediately after fetal delivery, intravenous infusion of $20 \mathrm{IU}$ oxytocin in $500 \mathrm{~mL}$ RL was started and transfused within 2 hours for women in both groups. Upon observing signs of placental separation (gush of blood from the vagina, descent of the umbilical cord, and an increase in the height of the uterus in the abdomen as the lower segment distends) and once the placenta came into the vagina, it was pulled out. The duration of the TSL was determined with a watch controlled by the same physician. Complete removal of the placenta and membranes was then confirmed. Any adverse event such as a prolonged third stage, a retained placenta, uterine atony, postpartum hemorrhage, and any vaginal tear was noted. If the placenta was not delivered after 30 minutes, it was considered a retained placenta. After completion of the TSL, the woman was transferred to the recovery room. Maternal pulse, blood pressure, and temperature were recorded within 15 minutes in the first hour and then hourly for the following six hours. At the postpartum sixth hour, a blood sample was taken to evaluate maternal hemoglobin levels.

All statistical analyses were performed using the Statistical Package for Social Sciences software, version 15.5 (SPSS, Chicago, IL, USA). Student's t-test and Mann-Whitney U test were used for statistical analyses. Parametric test results are presented as mean \pm standard deviation; nonparametric test results are presented as a median with minimum and maximum values. The probability of the null hypothesis was set to a 0.05 value. From a total of 112 women, data were analyzed from 53 women in the placental drainage with oxytocin group and from 59 women in the oxytocin alone group.

\section{Results}

Demographic characteristics of the women in the two groups were similar with regard to age, body mass index (BMI), gravidity, parity, weeks of gestation, and fetal birth weight; thus, these results demonstrate that the groups were similar to each other and comparable (Table 1). There was no significant difference between the groups with regard to the duration of the first and second stages of labor with $p$ values of 0.226 and 0.105 , respectively. However, the duration of the TSL was significantly shorter in the placenta drainage group than in the oxytocin alone group $(p<0.01)$ (Table 2$)$. In the oxytocin alone group, there was a single placental retention that was extracted with manual intervention under general anesthesia. 
Table 1: Demographic characteristics of the two groups

\begin{tabular}{|c|c|c|c|}
\hline Variables & Cord drainage $(n=53)$ & Oxytocin alone $(n=59)$ & $p$ value \\
\hline Age & $25.8 \pm 4.3$ & $27 \pm 5.4$ & $0.30^{a}$ \\
\hline BMI & $27.76 \pm 3.51$ & $28.17 \pm 3.57$ & $0.56^{a}$ \\
\hline Gravity & $2(1-10)$ & $2(1-5)$ & $0.79^{b}$ \\
\hline Parity & $1(0-4)$ & $1(1-3)$ & $0.37^{b}$ \\
\hline Gestational ages (weeks) & $39.3 \pm 1.3$ & $39 \pm 1.5$ & $0.29^{a}$ \\
\hline Birth weight (gram) & $3360 \pm 370.1$ & $3357.9 \pm 562.4$ & $0.92^{a}$ \\
\hline
\end{tabular}

Data presented as mean \pm standard deviation or median with minimum - maximum values

BMI: Body mass index, a: Student t test, b: Mann-Whitney U-test

Table 2: Comparison of duration of labor stages and hemoglobin values between the two groups

\begin{tabular}{lccr}
\hline Variables & Cord drainage $(\mathrm{n}=53)$ & Oxytocin alone $(\mathrm{n}=59)$ & $\mathrm{p}$ value \\
\hline Stage 1 (hour) & $8(2-25)$ & $8.5(3-22)$ & $0.27^{\mathrm{b}}$ \\
Stage 2 (minute) & $15(5-90)$ & $10(5-120)$ & $0.11^{\mathrm{b}}$ \\
Stage 3 (minute) & $3.4(0.35-16.2)$ & $5.10(2.3-11)$ & $<0.01^{\mathrm{b}}$ \\
Hemoglobin level on admission & $12.3 \pm 1.3$ & $12.3 \pm 1.1$ & $0.89^{\mathrm{a}}$ \\
Hemoglobin level at postpartum 6 & $11.6 \pm 1.2$ & $0,95^{\mathrm{a}}$ \\
Hemoglobin differences between on admission and & $11.6 \pm 1.3$ & & $0.17^{\mathrm{b}}$ \\
postpartum 6 & & $0.6(0.1-1.2)$ & \\
\hline
\end{tabular}

Data presented as mean \pm standard deviation or median with minimum - maximum values

a: Student $t$ test, $b$ : Mann-Whitney U-test

\section{Discussion}

The present study demonstrated that placental cord drainage shortens the duration of the third stage of labor in women who were administered an intravenous infusion of 20 IU oxytocin. Prolonged TSL is the major cause of maternal morbidity and mortality worldwide (12). When the TSL is prolonged, uterine contractile abilities may decrease and blood flow from uterine vessels to the placental bed continues. Inefficient uterine contraction may lead to excessive blood loss from the placental bed and result in PPH $(1,13)$. PPH is commonly defined as a blood loss of $500 \mathrm{~mL}$ or more within 24 hours of giving birth (14). Although it is almost entirely a preventable condition, PPH is the most serious complication in obstetric practices, occurs in approximately $4 \%$ of vaginal deliveries, and is the major cause of maternal deaths from hemorrhage during childbearing (16). Two thirds of PPH cases occur in women with no previously known risk factors. Thus, all pregnant women are considered to be at risk for this condition (17). The degree of blood loss associated with placental separation and delivery depends on how quickly the placenta separates from the uterine wall and how effectively the uterine muscle contracts around the placental bed and the uterine blood vessels (3). If the placenta is not delivered within 10 minutes, the risk of PPH increases (18). Therefore, any intervention for shortening the duration of the TSL and preventing complications becomes important. AMTSL decreases the duration of TSL, decreases maternal blood loss, decreases the likelihood of uterine atony, and reduces the incidence of $\mathrm{PPH}$ by approximately $65 \%$ when compared with expectant management $(1,2,3,19)$. Therefore, The International Federation of Gynecologists and Obstetricians, the International Confederation of Midwives, and the World Health Organization recommend the routine use of AMTSL for all vaginal singleton births (20).

Active management of the third stage of labor with uterotonic drugs for preventing complications during the TSL is recommended for all births (21). Oxytocin can be administered IV or intramuscularly (IM) with fewer side effects. Prophylactic oxytocin, at any dose, decreases the development of uterine atony, PPH, and the need for therapeutic uterotonic drugs (21). Therefore, oxytocin is universally recommended as the first-line uterotonic drug of choice for the prevention of uterine atony and related complications (8). Additionally, oxytocin has a short half-life ( 3 minutes) and using oxytocin during the first and second stages of labor has no effect on TSL. Therefore, AMTSL should be recommended for all women during vaginal delivery regardless of whether they received oxytocin or not during the first and second stages of labor (2). For this reason, in the present study, we did not regard the use of oxytocin in the first and second stages of labor.

Although oxytocin could be administrated IV/IM, the most efficient route of oxytocin administration is a slow IV infusion or a bolus infusion given over 1-2 minutes $(21,22)$. Oxytocin acts quickly and effectively with minimal adverse effects and can be used in all women, which makes it the main interven- 
tion for reducing PPH and is the first uterotonic drug option (23). The administration of oxytocin during the TSL reduces the risk of PPH by $40 \%$ (24). Immediately after fetal delivery, starting intravenous infusion of $20 \mathrm{IU}$ of oxytocin in $500 \mathrm{~mL}$ $\mathrm{RL}$ and given within 2 hours is the routine protocol in our institute for AMTSL. This routine protocol was administered to both groups so as not to sustain any adverse effects of not using an appropriate AMTSL. This may explain why PPH or uterine atony did not develop, and the median duration of the TSL was shorter in both groups than previously reported expectant management.

It has been reported that placental cord drainage shortens the duration of TSLs. Giacalone et al. reported that in their randomized control study comparing placental cord drainage with expectant delivery of the placenta, the median TSL duration was 8 minutes in the placental cord drainage group and 15 minutes in the control group (25). Roy et al. reported that placental blood drainage during vaginal delivery shortens the duration of TSL, reduces both the blood loss and the incidence of PPH (26). Shravage et al. reported in their randomized controlled study that the average TSL duration was significantly shorter in the placental cord drainage group than in the control group (17). Sharma et al. performed a randomized study in which placental cord drainage was compared with placental cord traction during cesarean sections (10). They found that placental cord drainage shortened the TSL duration and reduced the retained placenta rate. In another study, Sharma et al. compared placental cord drainage with controlled cord traction after the administration of $0.2 \mathrm{mg}$ of ergonovine with the delivery of the anterior shoulder and immediate cord clamping to compare PPH and TSL length. They reported that placental cord drainage significantly reduces the TSL duration in vaginal deliveries (11). A 2011 Cochrane review reported that placental cord drainage significantly shortens TSL and decreases the average blood loss. The authors' concluded that there was a small reduction in the TSL length and in the amount of blood loss when cord drainage was compared with no cord drainage (27). In the present study, placental cord drainage shortened the TSL duration. This result is in accordance with the results from previous studies.

Herman et al. ultrasonographically demonstrated that a retro-placental myometrial contraction is mandatory to produce shearing forces on the interface between the placenta and the myometrium to supply placental separation (28). Contractions occurring prior to delivery are insufficient to cause placental separation since, in the presence of the fetus, the myometrium is unable to achieve the necessary strain for placental separation $(5,28)$. Placental cord drainage is likely to decrease placental volume and surface area that might facilitate placental separation and uterine contraction, which results in a further increase of the separated area. This facilitates uterine contraction and further placental separation, resulting in early placental delivery and a shortening of the TSL duration.
This may explain why the TSL was shorter in the placental cord drainage group in the current study.

Placental retention is defined as the failure of placental delivery within 30 to 45 minutes and it usually requires intervention to assist delivery (18). Failure of the retro-placental myometrium contraction is the usual cause of a retained placenta. When the duration of a placental retention increases to 30 minutes, there is a greater risk of PPH (5). Placental cord drainage may facilitate uterine contractions and shorten the TSL. In the present study, there was no placental retention in the study group, and only one in the control group, which was expulsed with manual intervention under general anesthesia. This may explain why the placental retention rate was decreased in the placental cord drainage group in both the present study and previous studies.

Blood loss during TSL was not estimated in the present study. In previous studies, blood losses were estimated using difference methods. During blood collection at TSL, some amniotic fluid might enter the collection bag, or there may be an omission of blood that spattered on drapes and gowns. Thus, the measurement remains open to inaccuracies that can especially affect the measurement of lower amounts of blood loss (17). Additionally, three guidelines from the American College of Obstetrician and Gynecologists, the Royal College of Obstetrician and Gynecologists, and the Society of Obstetricians and Gynecologists of Canada comment on the unreliability of estimated blood loss, such as using a visible estimate or through the use of blood collection drapes (8). Instead of attempting blood collection to measure blood losses, we compared hemoglobin differences between the patient at admission and at their postpartum sixth hour. We know from previous studies that shortening the TSL duration also decreases blood loss.

The present study demonstrated that placental blood drainage with oxytocin shortened the TSL duration compared to oxytocin alone. Placental blood drainage is simple, does not introduce any additional cost, and can be performed by any birthing attendant. It might be used alone where uterotonic agents were not available or combined with oxytocin for AMTSL in low-risk vaginal deliveries. Because of the small sample size, this study is limited in its ability to derive a definitive result and generalize the results to the entire population. Furthermore, well-randomized studies with large sample sizes are needed to evaluate the effectiveness of placental cord drainage with IV oxytocin administration in the TSL.

\section{References}

1. Leduc D, Senikas V, Lalonde AB, Leduc D, Ballerman C, Biringer A, et al. Active management of the third stage of labour: prevention and treatment of postpartum hemorrhage: Clinical Practice Obstetrics Committee. Int J Gynaecol Obstet 2010;108(3):258-67. 
2. Sosa CG, Althabe F, Belizan JM, Buekens P. Use of oxytocin during early stages of labor and its effect on active management of third stage of labor. Am J Obstet Gynecol 2011;204 (3):238.e1-5.

3. Westhoff G, Cotter AM, Tolosa JE. Prophylactic oxytocin for the third stage of labour to prevent postpartum haemorrhage. Cochrane Database Syst Rev 2013;10:10: CD001808.

4. Rogers J, Wood J, McCandlish R, Ayers S, Truesdale A, Elbourne D. Active versus expectant management of third stage of labour: The Hinchingbrooke randomised controlled trial. Lancet 1998;351(9104):693-9.

5. Weeks AD. The retained placenta. Best Pract Res Clin Obstet Gynaecol 2008;22(6):1103-17.

6. Nankali A, Keshavarzi F, Fakheri T, Zare S, Rezaei M, Daeichin S. Effect of intraumbilical vein oxytocin injection on third stage of labor. Taiwan J Obstet Gynecol 2013;52(1):57-60.

7. Alkıș I, Karaman E, Han A, Ark HC, Büyükkaya B. The fertility sparing management of postpartum hemorrhage: A series of 47 cases of Bakri balloon tamponade. Taiwan J Obstet Gynecol 2015;54(3):232-5.

8. Dahlke JD, Mendez-Figueroa H, Maggio L, Hauspurg AK, Sperling JD, Chauhan SP, et al. Prevention and management of postpartum hemorrhage: a comparison of 4 national guidelines. Am J Obstet Gynecol 2015;213(1):76. e1-10.

9. Du Y, Ye M, Zheng F. Active management of the third stage of labor with and without controlled cord traction: a systematic review and meta-analysis of randomized controlled trials. Acta Obstet Gynecol Scand 2014;93(7):62633.

10. Sharma JB, Sharman WA, Newman MRB, Smith RJ. Evaluation of placental drainage at caesarean section as a method of placental delivery. J Obstet Gynaecol 1995; 15(4):237-9.

11. Sharma JB, Pundir P, Malhotra M, Arora R. Evaluation of placental drainage as a method of placental delivery in vaginal deliveries. Arch Gynecol Obstet 2005;271(4): 343-5.

12. Say L, Chou D, Gemmill A, Tunçalp Ö, Moller AB, Daniels J, et al. Global causes of maternal death: a WHO systematic analysis. Lancet Glob Health 2014;2(6):e32333.

13. Weeks A. The prevention and treatment of postpartum haemorrhage: what do we know, and where do we go to next? BJOG 2015;122(2):202-10.

14. Tunçalp O, Souza JP, Gülmezoglu M; World Health Organization. New WHO recommendations on prevention and treatment of postpartum hemorrhage. Int J Gynaecol Obstet 2013;123(3):254-6.

15. Carillo AP, Chandraharan E. Postpartum haemorrhage and haematological management. Obste Gynaecol Reproduc
Medic 2014;24(10):291-295.

16. Gohil JT, Tripathi B. A Study to Compare the Efficacy of Misoprostol, Oxytocin, Methyl-ergometrine and Ergometrine-Oxytocin in Reducing. Blood Loss in Active Management of 3rd Stage of Labor. J Obstet Gynaecol India 2011;61(4):408-12.

17. Shravage J C, Silpa PJ. Randomized controlled trial of placental blood drainage for the prevention of postpartum hemorrhage. J Obstet Gynecol India 2007;57(3):213-5.

18. Magann EF, Doherty DA, Briery CM, Niederhauser A, Morrison JC. Timing of placental delivery to prevent postpartum haemorrhage: lessons learned from an abandoned randomised clinical trial. Aust NZ J Obstet Gynaecol 2006;46:549-51.

19. Begley CM, Gyte GM, Devane D, McGuire W, Weeks A. Active versus expectant management for women in the third stage of labour. Cochrane Database Syst Rev 2011; 7:CD007412.

20. Stanton C, Armbruster D, Knight R, Ariawan I, Gbangbade S, Getachew A, et al. Use of active management of the third stage of labour in seven developing countries. Bull World Health Organ 2009; 87(3):207-15.

21. WHO Recommendations for the Prevention and Treatment of Postpartum Haemorrhage. Geneva: World Health Organization; 2012. (cited 2014 April 22). Available from apps.who.int/iris/bitstream

22. Davies GA, Tessier JL, Woodman MC, Lipson A, Hahn PM. Maternal hemodynamics after oxytocin bolus compared with infusion in the third stage of labor: a randomized controlled trial. Obstet Gynecol 2005;105(2):294-9.

23. de Castro Parreira MV, Gomes NC. Preventing postpartum haemorrhage: active management of the third stage of labour. J Clin Nurs 2013;22(23-24):3372-87.

24. Kahn S, Meyer A, Beste J. Prophylactic oxytocin: before or after placental delivery? J Fam Pract 2008;57 (12):817-8.

25. Giacalone PL, Vignal J, Daures JP, Boulot P, Hedon B, Laffargue F. A randomized evaluation of two techniques of management of third stage of labour in women at low risk of postpartum hemorrhage. Br J Obstet Gynaecol 2000;107(3):396-400.

26. Roy P, Sujatha MS, Bhandiwad A, Biswas B, Chatterjee A. Placental Blood Drainage as a Part of Active Management of Third Stage of Labour After Spontaneous Vaginal Delivery J Obstet Gynecol India 2016;66(Suppl 1):142-5.

27. Soltani H, Poulose TA, Hutchon DR. Placental cord drainage after vaginal delivery as part of the management of the third stage of labour. Cochrane Database Syst Rev. 2011:9:CD004665.

28. Herman A, Weinraub Z, Bukovsky I, Arieli S, Zabow P, Caspi E, et al. Dynamic ultrasonographic imaging of the third stage of labor: new perspectives into third stage mechanism. Am J Obstet Gynecol 1993;168(5):1496-99. 